\title{
Cocktail therapy with a combination of interferon, ribavirin and angiotensin-II type 1 receptor blocker attenuates murine liver fibrosis development
}

\author{
HITOSHI YOSHIJI, RYUICHI NOGUCHI, YASUHIDE IKENAKA, KOSUKE KAJI, YOSUKE AIHARA, \\ YUSAKU SHIRAI, JUNICHI YOSHII, KOJI YANASE and HIROSHI FUKUI
}

Third Department of Internal Medicine, Nara Medical University, Nara, Japan

Received January 17, 2011; Accepted March 3, 2011

DOI: $10.3892 / \mathrm{ijmm} .2011 .658$

\begin{abstract}
An effective therapeutic strategy for suppressing liver fibrosis development should improve the overall prognosis of patients with chronic liver diseases. Despite efforts to develop anti-fibrotic agents, no drugs have yet been approved as anti-fibrotic treatments for humans. An alternative strategy may be to employ a clinically available agent that also exhibits anti-fibrotic activities, for which the safety of long-term administration has been proven. The aim of the current study was to elucidate the combined effect of clinically used interferon (IFN), ribavirin (Rib) and angiotensin-II receptor blocker (ARB) on liver fibrosis development in mice. A model of $\mathrm{CCl}_{4}$-induced hepatic fibrosis was used to assess the effect of IFN, Rib and ARB. IFN, Rib and ARB were administered after a two-week treatment with $\mathrm{CCl}_{4}$, and the hepatic indices of fibrosis were assessed at eight weeks. Single treatment with IFN, Rib or ARB at the clinically available comparable doses significantly attenuated the liver fibrogenesis associated with the suppression of the number of $\alpha$-smooth muscle actin positive cells, and the hepatic transforming growth factor- $\beta$ (TGF- $\beta$ ) mRNA. Hepatic neovascularization, which is also known to play a pivotal role in liver fibrogenesis, and vascular endothelial growth factor (VEGF), a potent angiogenic factor, were also markedly inhibited. Combination treatment with any two agents exerted a more potent inhibitory effect than any single treatment. Moreover, the triple cocktail treatment revealed further suppressive effects than any two
\end{abstract}

Correspondence to: Dr Hitoshi Yoshiji, Third Department of Internal Medicine, Nara Medical University, Shijo-cho 840, Kashihara, Nara 634-8522, Japan

E-mail: yoshijih@naramed-u.ac.jp

Abbreviations: AT-II, angiotensin-II; ACE, angiotensin-converting enzyme; Ac-HSC, activated hepatic stellate cells; AT1-R, angiotensin type-1 receptor; ACE-I, ACE inhibitor; ARB, angiotensin type-1 receptor blocker; EC, endothelial cell; IFN, interferon; Rib, ribavirin; VEGF, vascular endothelial growth factor

Key words: interferon, ribavirin, angiotensin-II agent combination. Furthermore, in vitro studies showed that similar combined effects were observed on the proliferation and TGF- $\beta$ mRNA expression of activated hepatic stellate cells and endothelial cell tube formation. These results indicate that the cocktail combination treatment of clinically used IFN, Rib and ARB may provide a new strategy for anti-liver fibrosis therapy.

\section{Introduction}

Liver fibrosis is a characteristic feature of chronic liver diseases regardless of their etiology, such as hepatitis $\mathrm{B}$ (HBV) or hepatitis $\mathrm{C}(\mathrm{HCV})$. Cirrhosis can be defined as the end stage consequence of chronic liver diseases, and hepatic failure due to liver cirrhosis is caused by progressive fibrosis that ultimately results in nodular regeneration with loss of function (1-3). In human livers, fibrogenesis underlies the development of hepatocellular carcinoma (HCC) in at least $90 \%$ of cases and HCC is an ominous complication of cirrhosis in $30 \%$ of the patients (4). It has been reported that the risk of HCC increases in parallel with the progression of hepatic fibrosis (5). Furthermore, the existence of fibrosis itself accelerates experimental hepatocarcinogenesis (6). Taken together, an effective therapeutic strategy for suppressing liver fibrosis should improve the overall prognosis of patients with chronic liver diseases. Although emerging efforts are conducted to develop anti-fibrotic agents, no drugs have yet been approved as anti-fibrotic treatments for humans (3). Since liver fibrosis develops with chronic liver disease, therapies should be well tested and proven safe over decades, with good targeting to the liver and few adverse effects on other organs.

Chronic HCV infection is a major cause of liver fibrosis ultimately leading to cirrhosis (7). The combination treatment with pegylated interferon (Peg-IFN) and ribavirin (Rib) is widely used for the eradication of HCV. Therapy with Peg-IFN and Rib for 24 to 48 weeks leads to a sustained loss of $\mathrm{HCV}$ in the serum in approximately half of all patients (7). One approach to the management of chronic hepatitis $\mathrm{C}$ (CHC) in patients who do not have a sustained virological response to initial therapy is long-term maintenance therapy of IFN (8). In addition to the anti-viral effect, several lines of evidence suggest that IFNs are also effective in curtailing 
liver fibrosis both in experimental animal models and in clinical practice (9-12). A retrospective cohort study on patients with $\mathrm{CHC}$, in whom histological changes of hepatic fibrosis were confirmed, revealed that regression of fibrosis was associated with a sustained virological response to IFN (13). It has been reported that IFN therapy significantly suppressed hepatic stellate cell (HSC) activation in humans, and this reduction strongly correlated with an improvement in histological changes (14). It should be noted, however, that this histological improvement could be achieved only in virological responders. The overall rate of viral eradication by the current regimen of IFN is still not satisfactory, even if it is combined with an anti-viral agent, Rib (15). It has been reported that long-term administration of escalated doses of IFN increased the overall sustained virologic response rate, but, this regimen causes more frequent severe adverse effects, such as bone marrow suppression (12). Also, a recent large-scale clinical trial (HALT-C) has indicated that single treatment with IFN does not influence fibrosis progression (16). Alternative strategies such as a combination therapy with other safe agents in virological non-responders should be employed, since the combination treatment with different anti-fibrotic agents exerted a more potent inhibitory effect than any single agent (17).

It has been reported that the renin-angiotensin system (RAS) is activated in patients with chronic liver diseases, such as cirrhosis (18). Angiotensin-II (AT-II) has been shown to induce HSC contraction and proliferation, which play a pivotal role in liver fibrogenesis (19). We previously reported that the clinically used angiotensin-converting enzyme (ACE) inhibitor (ACE-I), and the angiotensin type-1 receptor (AT1-R) blockers (ARB), significantly attenuated experimental liver fibrosis development and suppressed activated HSCs (Ac-HSC) (20). We also observed that serum fibrosis markers significantly improved through treatment with a combination of low dose IFN and ACE-I in patients with CHC, despite their being refractory to IFN monotherapy (21). In addition, Rib has been reported to exert an anti-proliferative effect on HSCs (22).

Although previous studies conducted to determine the molecular processes associated with fibrosis and angiogenesis were performed independently, recent studies have revealed that both biological phenomena emerge synergistically $(23,24)$. We and other groups reported that neovascularization significantly increased during the liver fibrosis development (25-28). We previously reported that IFN and ARB exerted anti-angiogenic activities (29-31). In addition, a recent study has shown that Rib also exerted an anti-angiogenic activity in vitro (32).

In the current study, to improve the feasibility of a future clinical application, we examined the effects of a cocktail combination treatment involving IFN, Rib, and ARB at clinically comparable low doses on the development of murine liver fibrosis, and attempted to investigate the possible mechanisms involved, especially in conjunction with its effects on HSCs and angiogenesis.

\section{Materials and methods}

Animals and reagents. Male 6-week-old BALB/c mice, were purchased from Japan SLC Inc. (Shizuoka, Japan). The mice were housed in stainless-steel, mesh cages under controlled conditions of temperature and relative humidity $(50 \pm 20 \%)$, with 10-15 air changes per hour and $12 \mathrm{~h}$ /day light illumination. The animals were allowed access to food and tap water ad libitum throughout the acclimatization and experimental periods. The mouse IFN, Rib, and ARB (Losartan) were generously supplied by Dai-Nippon-Sumitomo Pharmaceutical Co. (Tokyo, Japan), and MSD Japan (Tokyo, Japan). AT-II was purchased from Wako Pure Chemical Industries (Tokyo, Japan).

Animal treatment. The mice were divided into nine groups ( $n=10$ in each group). All experimental groups (Groups 1-8, G1-G8) except for the negative control (G9) received $\mathrm{CCl}_{4}$ ( $2 \mathrm{ml} / \mathrm{kg}$ of body weight dissolved in corn oil) twice a week to induce liver fibrosis. After a two-week treatment with $\mathrm{CCl}_{4}$, administration of IFN, Rib, and ARB was initiated. The doses of IFN, Rib, and ARB were $1 \times 10^{4}$ IU twice a week via a $2 \mathrm{mg} / \mathrm{kg} /$ day subcutaneous injection, and 10 and $30 \mathrm{mg} / \mathrm{kg}$ daily gavage administration, respectively. The mice in the G1 group did not receive any additional treatment (control group). The mice in the G2, G3 and G4 groups received IFN, Rib, and ARB, respectively. The mice in G5-G7 received combination treatments with two agents (IFN+ARB, IFN+Rib, Rib+ARB), respectively. The mice in the $\mathrm{G} 8$ group received the triple combination of IFN, Rib, and ARB. The mice which received only corn oil served as the negative control group (G9). The doses of the employed agents in the current study are almost comparable to those used in the clinical practice as previously described $(33,34)$. After eight weeks of treatment with $\mathrm{CCl}_{4}$, all the mice were euthanized via anesthesia. All animal procedures were performed according to standard protocols and in accordance with the standard recommendations for the proper care and use of laboratory animals.

Histological and immunohistochemical analysis. In all experimental groups, 5- $\mu \mathrm{m}$ sections of formalin-fixed and paraffin-embedded livers were routinely processed for Sirius Red (S-R) staining in order to determine any liver fibrosis development. Immunohistochemical staining of $\alpha$-smooth muscle actin ( $\alpha$-SMA) was performed as previously described using paraffin-embedded sections with a primary anti- $\alpha$-SMA antibody (Dako, Kyoto, Japan) (35). Semi-quantitative analyses of fibrosis development and the immunopositive cell area were carried out with the Fuji-BAS 2000 image analyzing system (Fuji, Tokyo, Japan) in six ocular fields (x40 magnification) per specimen from 5 mice. The $\alpha$-SMA-positive vessels in the portal area were not counted as they were assumed to be hepatic arteries. Only the $\alpha$-SMA-positive cells in the sinusoidal lining were included in the image analysis.

RNA expression of transforming growth factor (TGF)- $\beta$ in the liver by real-time PCR. Total RNA was extracted from both liver tissue and isolated Ac-HSC using the RNeasy mini kit (Qiagen, Tokyo, Japan) according to the manufacturer's instructions. Total RNA ( $2 \mu \mathrm{g})$ from each sample was reversetranscribed to complementary DNA (cDNA) using the High Capacity RNA-to-cDNA kit (Applied Biosystems, Foster City, CA, USA) according to the manufacturer's instructions. The mRNA expression of TGF- $\beta$ from liver tissue was estimated 

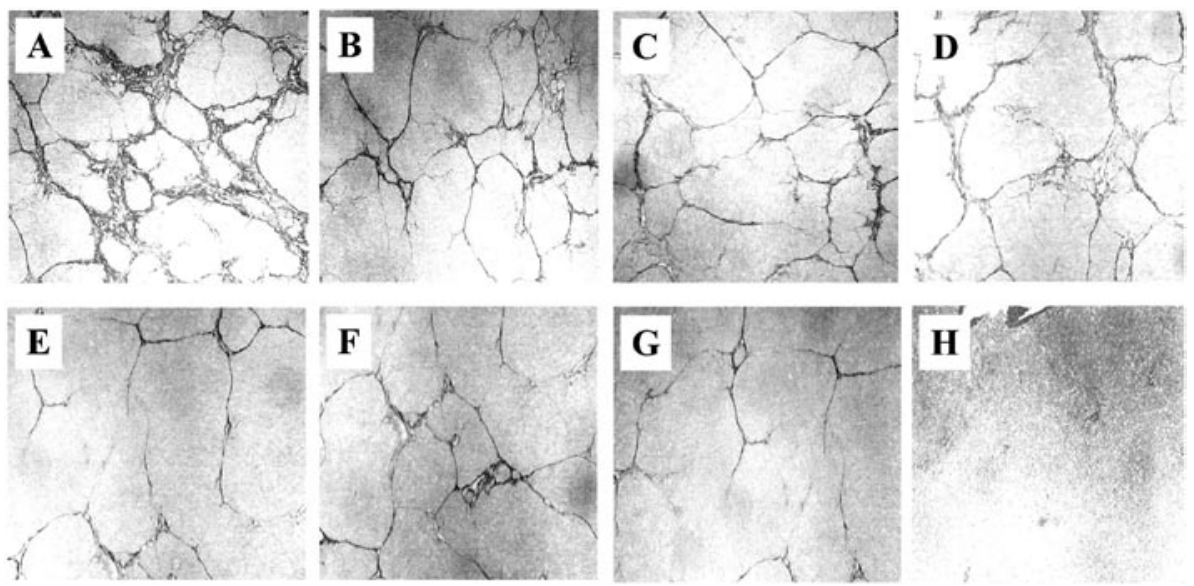

Figure 1. Microphotographs of liver sections from the $\mathrm{CCl}_{4}$-treated mice. (A) Control group after $\mathrm{CCl}_{4}$ treatment for 8 weeks. (B-D) IFN-, Rib-, and $\mathrm{ARB}$ treated groups, respectively. (E-G) Combined treatment with IFN+ARB, IFN+Rib, and ARB+Rib, respectively. (H) Triple combination treatment with IFN+Rib+ARB. No fibrosis development was found in the corn oil-treated group. $(\mathrm{x} 40)$.

with quantitative real-time PCR using the StepOnePlus real-time PCR system (Applied Biosystems) with the Fast SYBR-Green master mix (Applied Biosystems). The relative gene expression was measured using glyceraldehyde-3-phosphate dehydrogenase (GAPDH) as an internal control. The relative amount of target mRNA in each sample was determined by applying the threshold cycle to the standard curve.

In vitro assays. Primary HSCs were isolated from the liver of F344 rats, as previously described, with a minor modification (35). The cell viability was over $95 \%$, as determined by the trypan blue exclusion test. Freshly isolated HSCs were plated at a density of $5 \times 10^{5}$ cells $/ \mathrm{ml}$ on uncoated plastic dishes. After a 5-day culture, the HSCs became myofibroblast-like with reduced lipid vesicles and increased immunoreactive $\alpha$-SMA, and at 7 days after plating, all cells were well-spread and $\alpha$-SMA positive. From day 10 , the medium with or without treatment with IFN (100 IU/ml), Rib $(0.5 \mu \mathrm{g} / \mathrm{ml})$, or ARB $(10 \mu \mathrm{mol} / \mathrm{l})$ was changed every $24 \mathrm{~h}$ and cell culturing continued until day 12. The effects of IFN, Rib, and ARB on the proliferation of HSCs were determined by means of the tetrazolium, 3-(4,5-dimethylthiazol-2,5-yl)-2,5-diphenyltetrazolium bromide (MTT) assay as previously described (36). The in vitro angiogenesis was assessed as the formation of capillary-like structures of human umbilical vein endothelial cells co-cultured with human diploid fibroblasts as previously described (37). The experimental procedure followed the instructions provided with the angiogenesis kit (Kurabo, Tokyo, Japan). Computer-assisted quantitation of tubule formation was performed as in the in vivo assay.

Statistical analysis. To assess the statistical significance of the inter-group differences in the quantitative data, the Bonferroni's multiple comparisons test was used after a one-way ANOVA. This was followed by the Barlett's test to determine the homology of variance.

\section{Results}

Effects of IFN, Rib, and ARB on liver fibrosis development. S-R staining revealed that the 8-week treatment with $\mathrm{CCl}_{4}$

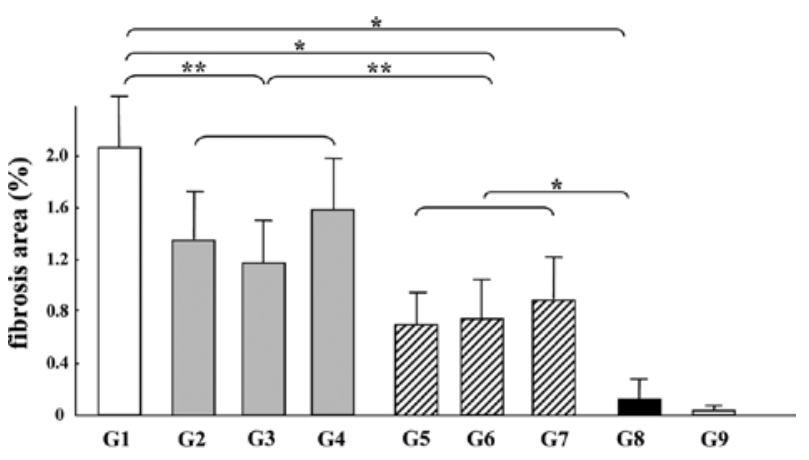

Figure 2. The effects of IFN, Rib and ARB on the fibrosis area. The fibrosis area was evaluated by an image analyzer as described in Materials and methods. Single treatment with IFN, Rib, or ARB suppressed fibrosis development. Combination treatment with any two agents exerted a more potent inhibitory effect than any single agent. However, the triple combination treatment with IFN, Rib, and ARB almost completely attenuated $\mathrm{CCl}_{4}$-induced liver fibrosis. No fibrosis development was found in the corn oil-treated group. G1, control group; G2-G4, IFN-, Rib-, and ARBtreated groups, respectively; G5-G7, combined treatments with IFN+ARB, IFN+Rib, and ARB+Rib, respectively; G8, triple combination treatment with IFN+Rib+ARB; G9, corn oil-treated negative control group. The data represent the means $\pm S D(n=10)$. *,**Statistically significant differences between the indicated groups $(\mathrm{p}<0.01$ and $\mathrm{p}<0.05$, respectively).

resulted in marked liver fibrosis development (Fig. 1A). Single treatment with IFN, Rib, or ARB suppressed fibrosis development (Fig. 1B-D, respectively). However, the combination treatments with any combination of the two agents exerted more potent inhibitory effects than the single agents (Fig. 1E-G). Furthermore, the triple combination treatment with IFN, Rib, and ARB almost completely attenuated the $\mathrm{CCl}_{4}$-induced liver fibrosis (Fig. $1 \mathrm{H}$ ). No fibrosis development was found in the corn oil-treated group (data not shown). As shown in Fig. 2, the densitometric analysis showed that the fibrosis areas closely corresponded to the histological findings. The semi-quantitative analysis also revealed that the triple combination treatment exerted the most potent inhibitory effect on liver fibrosis development. The serum ALT and total bilirubin levels were not significantly altered with the use of the agents, suggesting that the inhibitory effects of low doses of IFN, Rib, and ARB were not due to a non-specific 

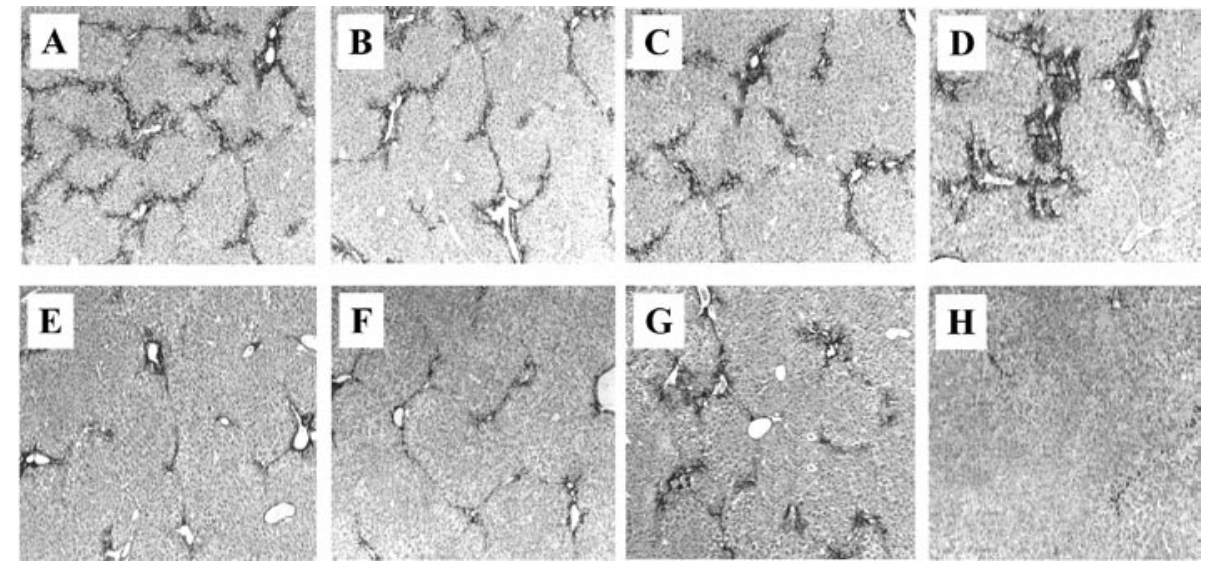

Figure 3. Immunohistochemical analysis of $\alpha$-smooth muscle actin ( $\alpha$-SMA). The immunopositive cells of $\alpha$-SMA were significantly reduced in number in the livers of the IFN-, Rib-, and ARB-treated groups, respectively (B-D) compared to the control group (A). (E-G) Combined treatments with IFN+ARB, IFN+Rib, and ARB+Rib, respectively. (H) Triple combination treatment with IFN+Rib+ARB. Magnification x40.

cytoprotective effect. The body weights and liver weights did not show any significant differences among the groups (data not shown).

Effects of IFN, Rib, and ARB on HSC activation in the liver. Immunohistochemical analysis of $\alpha$-SMA was carried out to examine the effects of IFN, Rib, and ARB on the activation of HSCs. The Ac-HSCs, which expressed $\alpha$-SMA, were reduced in number by treatment with IFN, Rib, and ARB (Fig. 3B-D) compared to the control group (Fig. 3A). Similarly to the anti-fibrotic effect, two-agent combination treatments induced further inhibition of the $\alpha$-SMA-positive cells compared to the administration of a single agent alone (Fig. 3E-G). Furthermore, the triple combination treatment with IFN, Rib, and ARB almost completely attenuated the $\alpha$-SMA-positive cells of the $\mathrm{CCl}_{4}$-induced liver fibrosis along with the liver fibrosis inhibition (Fig. 3H). Computer-assisted semi-quantitative analysis showed that the $\alpha$-SMA-positive cells in the IFN, Rib, and ARB-treated groups were significantly reduced almost in parallel with liver fibrosis development (Fig. 4A). We also performed real-time PCR analysis to elucidate the effect of these agents on TGF- $\beta$ mRNA expression. Similar to their effects on $\alpha$-SMA expression, IFN, Rib, and ARB also markedly suppressed the mRNA expression of TGF- $\beta$ in the liver (Fig. 4B). Noteworthy was the finding that the inhibitory effects of IFN, Rib, and ARB on $\alpha$-SMA and TGF- $\beta$ mRNA expressions, and those on the fibrosis area exerted almost parallel reductions.

Effects of IFN, Rib, and ARB on the cultured Ac-HSC. To elucidate whether IFN, Rib, and ARB directly affected Ac-HSC, we examined the effects of these agents on the proliferation of Ac-HSC in vitro. As shown in Fig. 5A, each single treatment suppressed the proliferation of Ac-HSC. Furthermore, treatment with any combination of the two agents exerted a more potent inhibitory effect than any single agent. However, the triple combination treatment with IFN, Rib, and ARB exerted the most potent inhibitory effect on the proliferation of HSC. We also examined the TGF- $\beta$ mRNA expression in the Ac-HSC. Similarly to the observed parallel reduction of $\alpha$-SMA-positive cells and TGF- $\beta$ mRNA expression in the

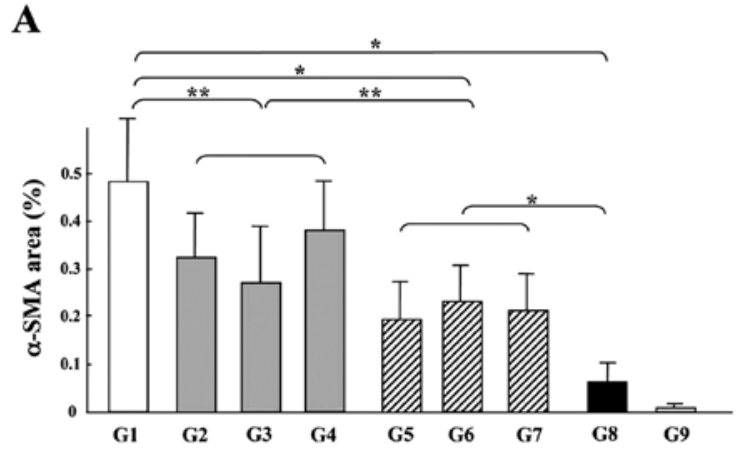

B

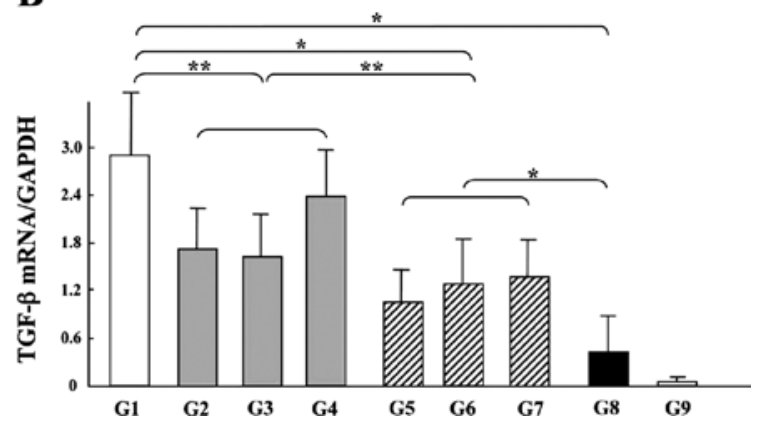

Figure 4. (A) Densitometric analysis of the $\alpha$-SMA-positive cells in the $\mathrm{CCl}_{4}$ treated liver. The $\alpha$-SMA-positive Ac-HSC were suppressed by treatment with IFN, Rib, or ARB (G2-G4), compared to the control group (G1). The combination treatment with any two agents exerted a more potent inhibitory effect than any single agent (G5-G7). However, the triple combination treatment with IFN, Rib, and ARB almost completely attenuated $\mathrm{CCl}_{4}$-induced liver fibrosis (G8). No marked $\alpha$-SMA-positive cells were observed in the corn oil-treated group (G9). (B) TGF- $\beta$ mRNA expression after treatment with IFN, Rib, or ARB. TGF- $\beta$ mRNA expression was evaluated by realtime PCR as described in Materials and methods. The inhibitory effects of IFN, Rib, or ARB on TGF- $\beta$ mRNA expression in the liver were almost in parallel with the suppression of $\alpha$-SMA-positive cells. G1, control group; G2-G4, IFN-, Rib-, and ARB-treated groups, respectively; G5-G7, combined treatments with IFN+ARB, IFN+Rib, and ARB+Rib, respectively; G8, triple combination treatment with IFN+Rib+ARB. G9, corn oil-treated negative control group. The data represent the mean \pm SD $(n=10) .{ }^{*} \mathrm{p}<0.01,{ }^{* *} \mathrm{p}<0.05$ indicate statistically significant differences between the groups.

liver, the IFN-, Rib-, and ARB-induced suppressive effects on HSC proliferation almost paralleled the reduction of TGF- $\beta$ mRNA expression (Fig. 5B). 
$\mathbf{A}$
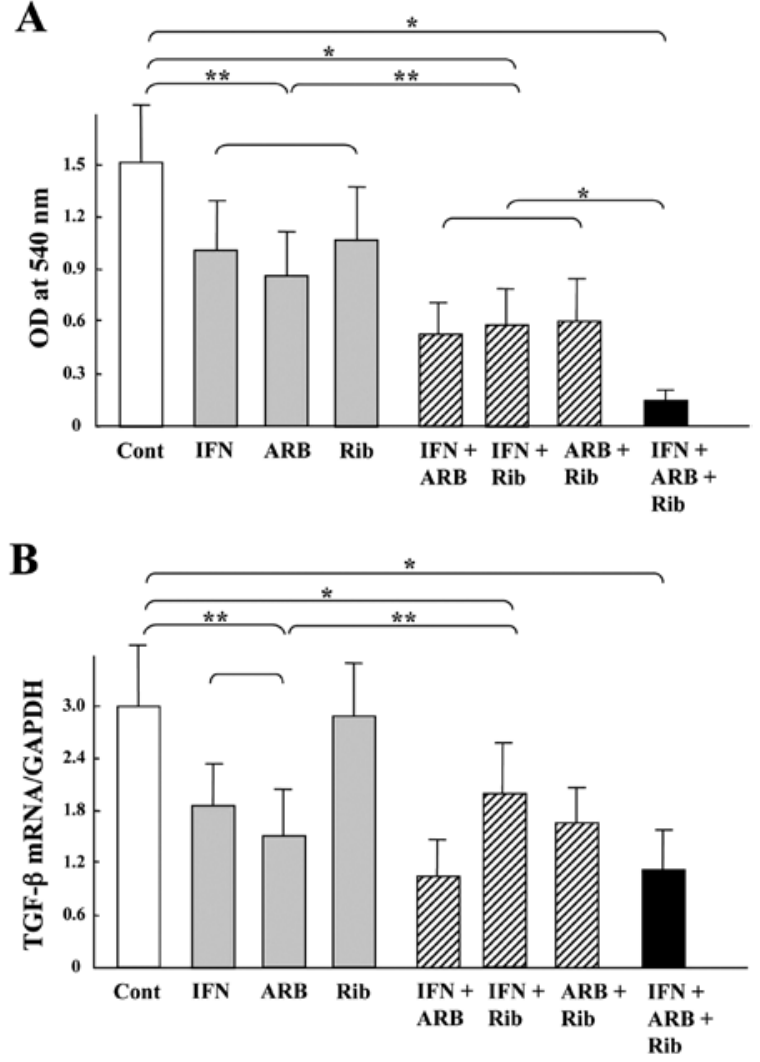

Figure 5. (A) Effects of IFN, Rib, or ARB on Ac-HSC proliferation in vitro. Cells were incubated in the presence of IFN (100 IU $/ \mathrm{ml})$, Rib $(0.5 \mu \mathrm{g} / \mathrm{ml})$, and ARB $(10 \mu \mathrm{mol} / \mathrm{l})$, and the proliferation was measured by the MTT assay as described in Materials and methods. Each single treatment suppressed the proliferation of HSC. Furthermore, the combination treatment with any two agents exerted a more potent inhibitory effect than any single agent. However, the triple combination treatment with IFN, Rib, and ARB exerted the most potent inhibitory effect on the proliferation of HSC. (B) Effects of IFN, Rib, or ARB on TGF- $\beta$ mRNA expression in activated HSC. The suppressive effect of the treatment with IFN, Rib, and ARB on HSC proliferation almost paralleled the reduction of TGF- $\beta$ mRNA expression. Cont, control group; IFN, ARB, Rib, single treatment with IFN, ARB, Rib, respectively. IFN+ARB, IFN+Rib, ARB+Rib, combination treatment with two agents simultaneously. IFN+ARB+Rib, triple combination treatment with IFN, Rib, and ARB. Each bar represents the means \pm SD $(n=5)$. ${ }^{*} p<0.05$ and ${ }^{* *} \mathrm{p}<0.01$ indicate statistically significant differences between the indicated experimental groups.

Effects of IFN, Rib, and ARB on hepatic neovascularization. To examine whether the inhibitory effects of IFN, Rib, and ARB were associated with the suppression of neovascularization in the liver, we evaluated the angiogenic response during liver fibrosis development. We performed a real-time PCR analysis of CD31 gene expression to evaluate neovascularization in the liver. We performed a preliminary immunohistochemical analysis of the von-Willebrand factor (vWF)-related antigen on sections from all experimental groups, and found that IFN, Rib, and ARB treatment significantly suppressed vWFpositive vessels. However, it was hard to accurately evaluate the vWF-positive cells because of difficulties in identifying the little slit vessels in the triple combination-treated group (data not shown). It has been reported that CD34 is a more sensitive marker than the vWF-related antigen (38). CD31 has also shown to be a sensitive marker in the endothelial cells (EC) (39). Among these markers, it has been reported that CD34 expression may be decreased by a potent angiogenic
A

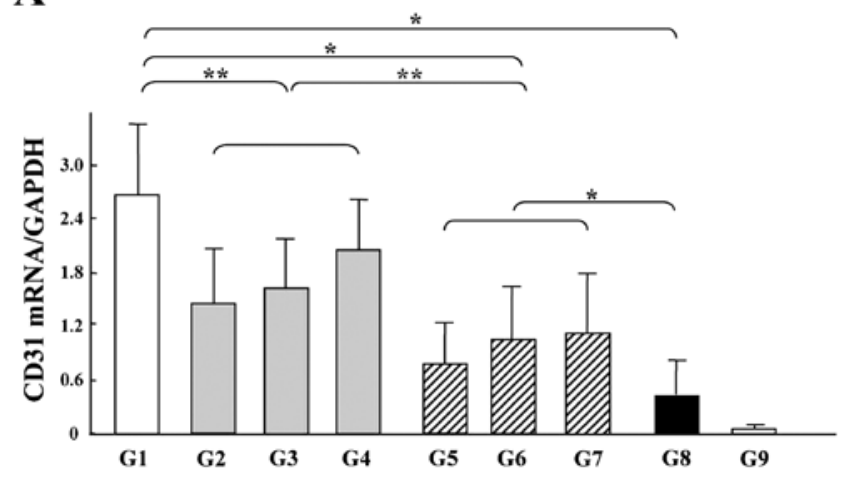

B

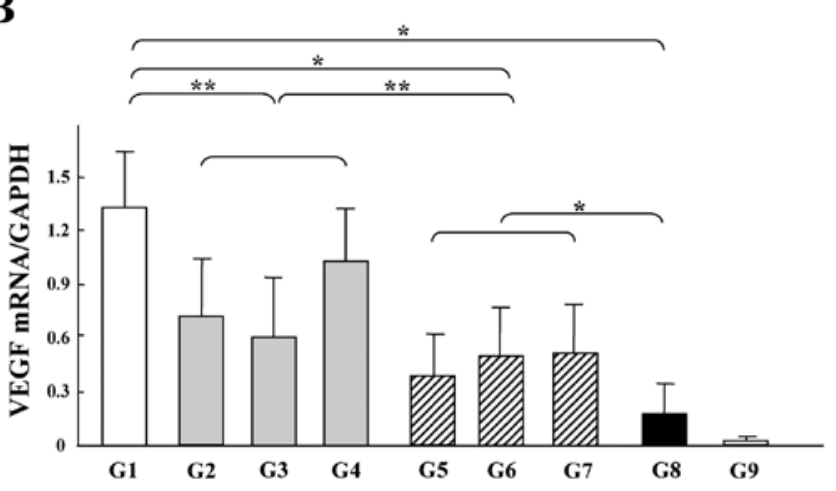

Figure 6. (A) Effects of IFN, Rib, or ARB on hepatic neovascularization. IFN, Rib, and ARB suppressed CD31 gene expression compared to the control group. Furthermore, the combination treatment with any two agents exerted a more potent inhibitory effect than any single agent. However, the triple combination treatment with IFN, Rib, and ARB exerted the most potent inhibitory effect on the neovascularization in the liver. (B) Effects of IFN, Rib, or ARB on the VEGF expression in the liver. VEGF expression in the liver was suppressed by treatment with IFN, Rib, and ARB, which almost matched the results of fibrosis development and neovascularization. G1, control group; G2-G4, IFN-, Rib-, and ARB-treated groups, respectively; G5-G7, combined treatment with IFN+ARB, IFN+Rib, and ARB+Rib, respectively; G8, triple combination treatment with IFN+Rib+ARB. Corn oil-treated negative control group (G9). The data represent the means $\pm \mathrm{SD}$ $(n=10) .{ }^{* * * *}$ Statistically significant differences between the indicated groups $(\mathrm{p}<0.01$ and $\mathrm{p}<0.05$, respectively).

factor, the vascular endothelial growth factor (VEGF) (40). We thus used CD31 expression in the current study. We performed a real-time PCR analysis of CD31 gene expression to evaluate neovascularization in the liver. Similar to the fibrosis area, IFN, Rib, and ARB suppressed CD31 gene expression compared to the control group. Furthermore, the combination treatment with any two agents exerted a more potent inhibitory effect than any single agent. However, the triple combination treatment with IFN, Rib, and ARB exerted the most potent inhibitory effect on neovascularization in the liver (Fig. 6A). Noteworthy was the finding that the suppression of angiogenesis by treatment with IFN, Rib, and ARB was of a similar magnitude to that of the inhibition of the fibrosis areas. To elucidate whether or not the suppressive effects of IFN, $\mathrm{Rib}$, and ARB on neovascularization were accompanied with inhibition of VEGF, we examined the VEGF expression in the liver. As shown in Fig. 6B, the VEGF expression in the liver was suppressed by treatment with IFN, Rib, and ARB, which almost matched the results of fibrosis development and neovascularization. 
A
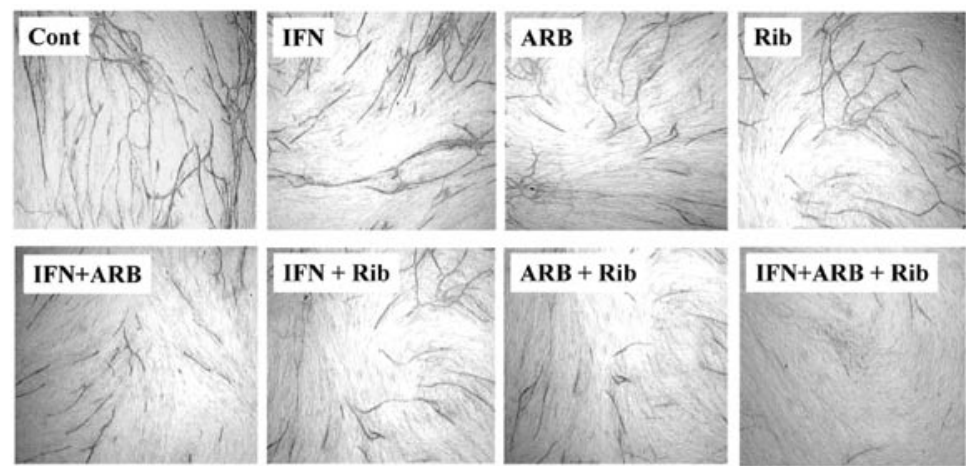

B

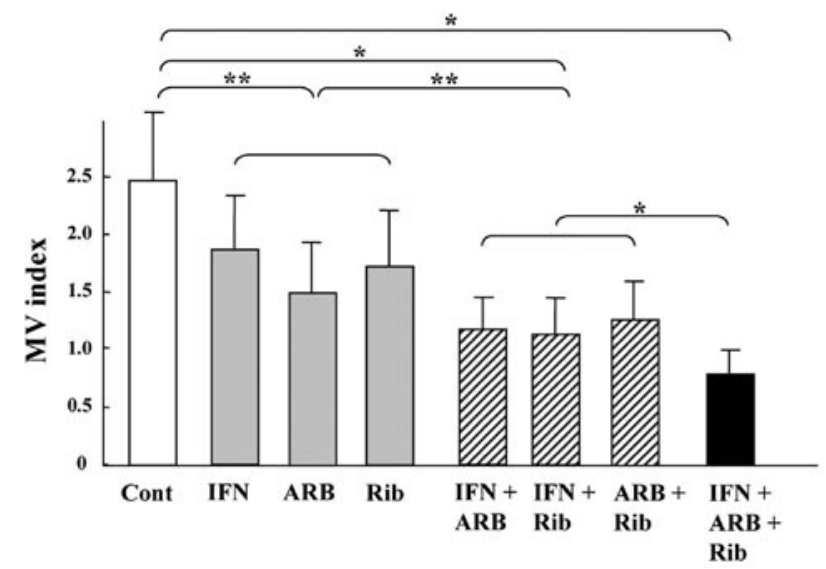

Figure 7. Effects of IFN, Rib, or ARB on endothelial cell (EC) tubular formation. (A) Representative features of in vitro EC tubular formation. (B) The quantitative analysis of the total length of the tubules formed in the IFN-, Rib- or ARB-treated cultures confirmed that the triple combination treatment almost completely attenuated EC tubular formation. Cont, control group; IFN, ARB, Rib, single treatments with IFN, ARB, Rib, respectively. IFN+ARB, IFN+Rib, $\mathrm{ARB}+\mathrm{Rib}$, combination treatments with two agents simultaneously. IFN+ARB+Rib, triple combination treatment with IFN, Rib, and ARB. Each bar represents the mean $\pm \mathrm{SD}(\mathrm{n}=5) .{ }^{*} \mathrm{p}<0.05$ and ${ }^{* *} \mathrm{p}<0.01$ indicate statistically significant differences between the indicated experimental groups.

Effect of IFN, Rib, and ARB on in vitro angiogenesis. To elucidate the direct effect of IFN, Rib, and ARB on angiogenesis, we next performed a set of in vitro experiments. Single treatment with each agent suppressed EC tubular formation, and the combination treatment of two agents exerted further inhibitory effects compared with each single treatment. Similarly to the effect on hepatic neovascularization, the triple combination treatment with IFN, Rib, and ARB exerted the most potent inhibitory effect on EC tubular formation (Fig. 7A). The quantitative analysis of the total length of the tubules formed in IFN-, Rib- or ARB-treated cultures confirmed that the triple combination treatment almost completely attenuated EC tubular formation (Fig. 7B). To examine whether the initial concentration of EC can influence the effect of these agents on tubular formation, we also examined EC tubular formation with different initial concentrations of $\mathrm{EC}$ on Matrige $\mathrm{l}^{\mathrm{TM}}$ as previously described (30). We found that IFN, Rib, and ARB suppressed EC tubular formation at a similar magnitude regardless of the initial EC concentrations (data not shown).

\section{Discussion}

Since it is now widely recognized that many factors are involved in the development of liver fibrogenesis, combination therapy of agents with different mechanisms of action should be better than any single agent treatment. To examine the feasibility of the future clinical application of a combination treatment, we employed clinically used agents, but not newly developing modalities such as gene therapy. In the current study, we demonstrated that IFN, Rib, and ARB suppressed liver fibrosis development and also suppressed HSC activation and neovascularization. The combination treatment with any two agents exerted a more potent inhibitory effect than any single agent. However, the triple combination treatment with IFN, Rib, and ARB almost completely attenuated $\mathrm{CCl}_{4}$-induced liver fibrosis. It has been reported that treatment with IFN at $1 \times 10^{6} \mathrm{U} / \mathrm{kg}$ in mice is almost equal to $5.5 \times 10^{6}$ units every day in humans (41). In the current study, we administered IFN at a dose of $1 \times 10^{4}$ units per mouse twice a week. Based on this conversion, our dosage generally equaled $2 \times 10^{6}$ units in humans. Since the usual clinical dose of IFN for chronic hepatitis is $3-6 \times 10^{6}$ units three times a week, we assume that the dose in our current study was comparable to a relatively low dose in clinical practice.

It is now widely recognized that Ac-HSC plays a pivotal role in liver fibrosis development $(42,43)$. The increased number of HSCs during liver fibrogenesis reflects the activity of several growth factors (42). Among the growth factors, TGF- $\beta$ is one of the key mediators in liver fibrogenesis $(3,44)$. Liver fibrosis is characterized by excessive deposition of extracellular matrix (ECM), which leads to severe pathological disturbance in the liver. TGF- $\beta$ is an important cytokine in the regulation of ECM production. Strategies aiming at disrupting TGF- $\beta$ synthesis and signaling cascade markedly suppress liver fibrosis development. Animal and culture studies using soluble TGF- $\beta$ receptors or other means such as neutralizing 
monoclonal antibodies and dominant negative receptor gene transfer have shown promising results (45-47).

It has been reported that IFN down-regulated collagen promoter activity and antagonized TGF- $\beta$-stimulated collagen gene transcription in Ac-HSC. The inhibitory effect of IFN- $\alpha$ on collagen transcription was exerted through interaction between phosphorylated Stat1 and p300. AT-II is also a potent inducer of TGF- $\beta$ synthesis through Smad 2 in cultured HSC in vitro $(48,49)$. AT-II induces HSC proliferation and up-regulates TGF- $\beta$ expression via the AT1-R pathway (20). The increase of the hepatic concentration of TGF- $\beta$ induced by bile-duct ligation was attenuated in the AT1-R knockout mice (50). In humans, it has been shown that a combination of AT-II and TGF- $\beta$ genotypic polymorphism is associated with advanced hepatic fibrosis (51). In Ac-HSC, AT-II phosphorylated Akt and MAPKs, and increased AP-1 binding in a redox sensitive manner, suggest that the main signaling cascades of IFN and AT-II seem to be different $(48,52)$. In the current study, contrary to IFN and ARB, Rib did not suppress TGF- $\beta$ expression in activated HSCs, whereas proliferation was significantly inhibited. Rib decreased HSC proliferation by inhibiting the p-c-Jun signaling cascade (22). Collectively, these different biological activities of IFN, Rib, and ARB on Ac-HSC contribute to the combined inhibitory effects of these agents on liver fibrogenesis.

Emerging evidence from recent studies indicates that angiogenesis plays a pivotal role in many physiological and pathological processes, such as tumor growth, arthritis, psoriasis, and diabetic retinopathy $(53,54)$. Although previous studies conducted to determine the molecular processes associated with fibrosis and angiogenesis were performed independently, several recent studies have revealed that both biological phenomena emerge synergistically (55). We previously reported that neovascularization significantly increased during the development of liver fibrosis, and that suppression of angiogenic signaling markedly attenuated liver fibrogenesis (25).

Among the identified angiogenic factors to date, VEGF is one of the most potent and central factors in many physiological and pathological processes (56-58). In liver fibrosis, it has been shown that VEGF expression increased both in human chronic liver diseases and in experimental fibrogenesis (59-61). It has also been reported that VEGF expression correlates with chronic liver disease-associated angiogenesis and sinusoidal capillarization $(59,62,63)$. We also observed that VEGF gene expression significantly increased during fibrosis development associated with neovascularization in the liver, and that the suppression of VEGF-receptor interaction significantly attenuated the progression of liver fibrosis and angiogenesis (25). In addition to EC, the expression of VEGF and its receptor occurs in activated HSCs, indicating that the combined effect of VEGF both on HSCs and ECs plays an important role in liver fibrosis development. We previously reported that IFN and ARB significantly suppressed VEGFmediated neovascularization in tumors $(29,64)$. In the current study, we also found that these agents markedly attenuated hepatic neovascularization during liver fibrosis development. Similarly to the action on Ac-HSC, the inhibitory effect of Rib on angiogenesis mediated a different cascade from the other two agents via the suppression of tetrahydrobiopterin
(32). As per the effects on Ac-HSC, the different biological activities of IFN, Rib, and ARB augment the combined inhibitory effects. Further studies are required to elucidate the exact mechanistic insights.

In summary, we have shown that combined treatment with IFN, Rib, and ARB at clinically comparable low doses significantly inhibited liver fibrogenesis, at least partly, via suppression of activated HSC and neovascularization, respectively. Since these three agents are already widely used in clinical practice, it may represent a potential new therapeutic strategy against the progression of liver fibrosis development in the future.

\section{Acknowledgements}

This study was supported in part by a Grant-in-Aid from the Ministry of Health, Labor and Welfare, Japan.

\section{References}

1. Albanis E and Friedman SL: Hepatic fibrosis. Pathogenesis and principles of therapy. Clin Liver Dis 5: 315-334, v-vi, 2001.

2. Olaso E and Friedman SL: Molecular regulation of hepatic fibrogenesis. J Hepatol 29: 836-847, 1998.

3. Friedman SL: Liver fibrosis - from bench to bedside. J Hepatol 38 (Suppl. 1): S38-S53, 2003.

4. Befeler AS and Di Bisceglie AM: Hepatocellular carcinoma: diagnosis and treatment. Gastroenterology 122: 1609-1619, 2002 .

5. Okita K, Sakaida I and Hino K: Current strategies for chemoprevention of hepatocellular carcinoma. Oncology 62 (Suppl. 1): 24-28, 2002.

6. Sakaida I, Hironaka K, Uchida K, Suzuki C, Kayano K and Okita K: Fibrosis accelerates the development of enzyme-altered lesions in the rat liver. Hepatology 28: 1247-1252, 1998.

7. Ghany MG, Strader DB, Thomas DL and Seeff LB: Diagnosis, management, and treatment of hepatitis C: an update. Hepatology 49: 1335-1374, 2009.

8. Shiffman ML: Impact of peginterferon maintenance therapy on the risk of developing hepatocellular carcinoma in patients with chronic hepatitis C virus. Oncology 78 (Suppl. 1): 11-16, 2010.

9. Fort J, Pilette C, Veal N, et al: Effects of long-term administration of interferon alpha in two models of liver fibrosis in rats. J Hepatol 29: 263-270, 1998.

10. Okanoue T, Itoh Y, Minami M, et al: Interferon therapy lowers the rate of progression to hepatocellular carcinoma in chronic hepatitis C, but not significantly in an advanced stage: a retrospective study in 1148 patients. Viral Hepatitis Therapy Study Group. J Hepatol 30: 653-659, 1999.

11. Poynard T, Leroy V, Cohard M, et al: Meta-analysis of interferon randomized trials in the treatment of viral hepatitis $C$ : effects of dose and duration. Hepatology 24: 778-789, 1996.

12. Suzuki K, Aoki K, Ohnami S, et al: Adenovirus-mediated gene transfer of interferon alpha improves dimethylnitrosamineinduced liver cirrhosis in rat model. Gene Ther 10: 765-773, 2003.

13. Shiratori Y, Imazeki F, Moriyama M, et al: Histologic improvement of fibrosis in patients with hepatitis $C$ who have sustained response to interferon therapy. Ann Intern Med 132: 517-524, 2000.

14. Guido M, Rugge M, Chemello L, et al: Liver stellate cells in chronic viral hepatitis: the effect of interferon therapy. J Hepatol 24: 301-307, 1996.

15. Arif A, Levine RA, Sanderson SO, et al: Regression of fibrosis in chronic hepatitis $\mathrm{C}$ after therapy with interferon and ribavirin. Dig Dis Sci 48: 1425-1430, 2003.

16. Di Bisceglie AM, Shiffman ML, Everson GT, et al: Prolonged therapy of advanced chronic hepatitis $\mathrm{C}$ with low-dose peginterferon. N Engl J Med 359: 2429-2441, 2008.

17. Yoshiji H, Noguchi R and Fukui $\mathrm{H}$ : Combined effect of an ACE inhibitor, perindopril, and interferon on liver fibrosis markers in patients with chronic hepatitis C. J Gastroenterol 40: 215-216, 2005. 
18. Helmy A, Jalan R, Newby DE, Hayes PC and Webb DJ: Role of angiotensin II in regulation of basal and sympathetically stimulated vascular tone in early and advanced cirrhosis. Gastroenterology 118: 565-572, 2000.

19. Bataller R, Gines P, Nicolas JM, et al: Angiotensin II induces contraction and proliferation of human hepatic stellate cells. Gastroenterology 118: 1149-1156, 2000.

20. Yoshiji H, Kuriyama S, Yoshii J, et al: Angiotensin-II type 1 receptor interaction is a major regulator for liver fibrosis development in rats. Hepatology 34: 745-750, 2001.

21. Yoshiji H, Noguchi R, Kojima H, et al: Interferon augments the anti-fibrotic activity of an angiotensin-converting enzyme inhibitor in patients with refractory chronic hepatitis C. World J Gastroenterol 12: 6786-6791, 2006.

22. Khan F, Peltekian KM and Peterson TC: Effect of interferonalpha, ribavirin, pentoxifylline, and interleukin-18 antibody on hepatitis C sera-stimulated hepatic stellate cell proliferation. J Interferon Cytokine Res 28: 643-651, 2008.

23. Paternostro C, David E, Novo E and Parola M: Hypoxia, angiogenesis and liver fibrogenesis in the progression of chronic liver diseases. World J Gastroenterol 16: 281-288, 2010.

24. Valfre di Bonzo L, Novo E, Cannito S, et al: Angiogenesis and liver fibrogenesis. Histol Histopathol 24: 1323-1341, 2009.

25. Yoshiji H, Kuriyama S, Yoshii J, et al: Vascular endothelial growth factor and receptor interaction is a prerequisite for murine hepatic fibrogenesis. Gut 52: 1347-1354, 2003.

26. Kitade M, Yoshiji H, Kojima H, et al: Leptin-mediated neovascularization is a prerequisite for progression of nonalcoholic steatohepatitis in rats. Hepatology 44: 983-991, 2006.

27. Amarapurkar AD, Amarapurkar DN, Vibhav S and Patel ND: Angiogenesis in chronic liver disease. Ann Hepatol 6: 170-173, 2007.

28. Ueno T, Nakamura T, Torimura T and Sata M: Angiogenic cell therapy for hepatic fibrosis. Med Mol Morphol 39: 16-21, 2006.

29. Noguchi R, Yoshiji H, Kuriyama S, et al: Combination of interferon-beta and the angiotensin-converting enzyme inhibitor, perindopril, attenuates murine hepatocellular carcinoma development and angiogenesis. Clin Cancer Res 9: 6038-6045, 2003.

30. Yoshiji H, Kuriyama S, Kawata M, et al: The angiotensin-Iconverting enzyme inhibitor perindopril suppresses tumor growth and angiogenesis: possible role of the vascular endothelial growth factor. Clin Cancer Res 7: 1073-1078, 2001.

31. Yoshiji H, Noguchi R, Ikenaka Y, et al: Renin-angiotensin system inhibitors as therapeutic alternatives in the treatment of chronic liver diseases. Curr Med Chem 14: 2749-2754, 2007.

32. Michaelis M, Michaelis R, Suhan T, et al: Ribavirin inhibits angiogenesis by tetrahydrobiopterin depletion. FASEB J 21 : 81-87, 2007.

33. Yoshiji H, Kuriyama S and Fukui H: Angiotensin-I-converting enzyme inhibitors may be an alternative anti-angiogenic strategy in the treatment of liver fibrosis and hepatocellular carcinoma Possible role of vascular endothelial growth factor. Tumour Biol 23: 348-356, 2002

34. Yoshiji H, Noguchi R, Ikenaka Y, et al: Losartan, an angiotensin-II type 1 receptor blocker, attenuates the liver fibrosis development of non-alcoholic steatohepatitis in the rat. BMC Res Notes 2: 70, 2009.

35. Yoshiji H, Kuriyama S, Miyamoto Y, et al: Tissue inhibitor of metalloproteinases-1 promotes liver fibrosis development in a transgenic mouse model. Hepatology 32: 1248-1254, 2000.

36. Yoshiji H, Kuriyama S, Noguchi R, et al: Angiopoietin 2 displays a vascular endothelial growth factor dependent synergistic effect in hepatocellular carcinoma development in mice. Gut 54: $1768-1775,2005$

37. Yoshiji H, Noguchi R, Kaji K, et al: Attenuation of insulinresistance-based hepatocarcinogenesis and angiogenesis by combined treatment with branched-chain amino acids and angiotensin-converting enzyme inhibitor in obese diabetic rats. J Gastroenterol 45: 443-450, 2010.

38. Tanigawa N, Lu C, Mitsui T and Miura S: Quantitation of sinusoid-like vessels in hepatocellular carcinoma: its clinical and prognostic significance. Hepatology 26: 1216-1223, 1997.

39. Lindsay CK, Sinha CC and Thorgeirsson UP: Morphological study of vascular dissemination in a metastatic hepatocellular carcinoma model in the monkey. Hepatology 26: 1209-1215, 1997.

40. Hellwig SM, Damen CA, van Adrichem NP, Blijham GH, Groenewegen G and Griffioen AW: Endothelial CD34 is suppressed in human malignancies: role of angiogenic factors. Cancer Lett 120: 203-211, 1997.
41. Wang L, Tang ZY, Qin LX, et al: High-dose and long-term therapy with interferon-alfa inhibits tumor growth and recurrence in nude mice bearing human hepatocellular carcinoma xenografts with high metastatic potential. Hepatology 32: 43-48, 2000.

42. Pinzani $M$ and Marra F: Cytokine receptors and signaling in hepatic stellate cells. Semin Liver Dis 21: 397-416, 2001.

43. Friedman SL: Cytokines and fibrogenesis. Semin Liver Dis 19: 129-140, 1999.

44. Bataller R, Gabele E, Parsons CJ, et al: Systemic infusion of angiotensin II exacerbates liver fibrosis in bile duct-ligated rats. Hepatology 41: 1046-1055, 2005.

45. Arias M, Lahme B, Van de Leur E, Gressner AM and Weiskirchen R: Adenoviral delivery of an antisense RNA complementary to the $3^{\prime}$ coding sequence of transforming growth factor-betal inhibits fibrogenic activities of hepatic stellate cells. Cell Growth Differ 13: 265-273, 2002.

46. George J, Roulot D, Koteliansky VE and Bissell DM: In vivo inhibition of rat stellate cell activation by soluble transforming growth factor beta type II receptor: a potential new therapy for hepatic fibrosis. Proc Natl Acad Sci USA 96: 12719-12724, 1999.

47. Nakamura T, Sakata R, Ueno T, Sata M and Ueno H: Inhibition of transforming growth factor beta prevents progression of liver fibrosis and enhances hepatocyte regeneration in dimethylnitrosamine-treated rats. Hepatology 32: 247-255, 2000.

48. Bataller R, Sancho-Bru P, Gines P, et al: Activated human hepatic stellate cells express the renin-angiotensin system and synthesize angiotensin II. Gastroenterology 125: 117-125, 2003.

49. Kamada Y, Tamura S, Kiso S, et al: Angiotensin II stimulates the nuclear translocation of Smad2 and induces PAI-1 mRNA in rat hepatic stellate cells. Hepatol Res 25: 296-305, 2003.

50. Yang L, Bataller R, Dulyx J, et al: Attenuated hepatic inflammation and fibrosis in angiotensin type 1a receptor deficient mice. J Hepatol 43: 317-323, 2005.

51. Dixon JB, Bhathal PS, Jonsson JR, Dixon AF, Powell EE and O'Brien PE: Pro-fibrotic polymorphisms predictive of advanced liver fibrosis in the severely obese. J Hepatol 39: 967-971, 2003.

52. Inagaki $\mathrm{Y}$, Nemoto $\mathrm{T}$, Kushida $\mathrm{M}$, et al: Interferon alfa down-regulates collagen gene transcription and suppresses experimental hepatic fibrosis in mice. Hepatology 38: 890-899, 2003.

53. Carmeliet P: Mechanisms of angiogenesis and arteriogenesis. Nat Med 6: 389-395, 2000.

54. Carmeliet P and Jain RK: Angiogenesis in cancer and other diseases. Nature 407: 249-257, 2000.

55. Kalluri R and Sukhatme VP: Fibrosis and angiogenesis. Curr Opin Nephrol Hypertens 9: 413-418, 2000.

56. Karkkainen MJ and Petrova TV: Vascular endothelial growth factor receptors in the regulation of angiogenesis and lymphangiogenesis. Oncogene 19: 5598-5605, 2000.

57. Yancopoulos GD, Davis S, Gale NW, Rudge JS, Wiegand SJ and Holash J: Vascular-specific growth factors and blood vessel formation. Nature 407: 242-248, 2000

58. Shibuya M: Structure and function of VEGF/VEGF-receptor system involved in angiogenesis. Cell Struct Funct 26: 25-35, 2001.

59. Rosmorduc O, Wendum D, Corpechot C, et al: Hepatocellular hypoxia-induced vascular endothelial growth factor expression and angiogenesis in experimental biliary cirrhosis. Am J Pathol 155: 1065-1073, 1999 .

60. Ohmori S, Shiraki K, Sugimoto K, et al: High expression of CD34-positive sinusoidal endothelial cells is a risk factor for hepatocellular carcinoma in patients with $\mathrm{HCV}$-associated chronic liver diseases. Hum Pathol 32: 1363-1370, 2001.

61. Corpechot C, Barbu V, Wendum D, et al: Hypoxia-induced VEGF and collagen I expressions are associated with angiogenesis and fibrogenesis in experimental cirrhosis. Hepatology 35: 1010-1021, 2002

62. Cui S, Hano H, Sakata A, et al: Enhanced CD34 expression of sinusoid-like vascular endothelial cells in hepatocellular carcinoma. Pathol Int 46: 751-756, 1996

63. El-Assal ON, Yamanoi A, Soda Y, et al: Clinical significance of microvessel density and vascular endothelial growth factor expression in hepatocellular carcinoma and surrounding liver: possible involvement of vascular endothelial growth factor in the angiogenesis of cirrhotic liver. Hepatology 27: 1554-1562, 1998.

64. Yoshiji H, Noguchi R, Kuriyama S, Yoshii J and Ikenaka Y: Combination of interferon and angiotensin-converting enzyme inhibitor, perindopril, suppresses liver carcinogenesis and angiogenesis in mice. Oncol Rep 13: 491-495, 2005. 